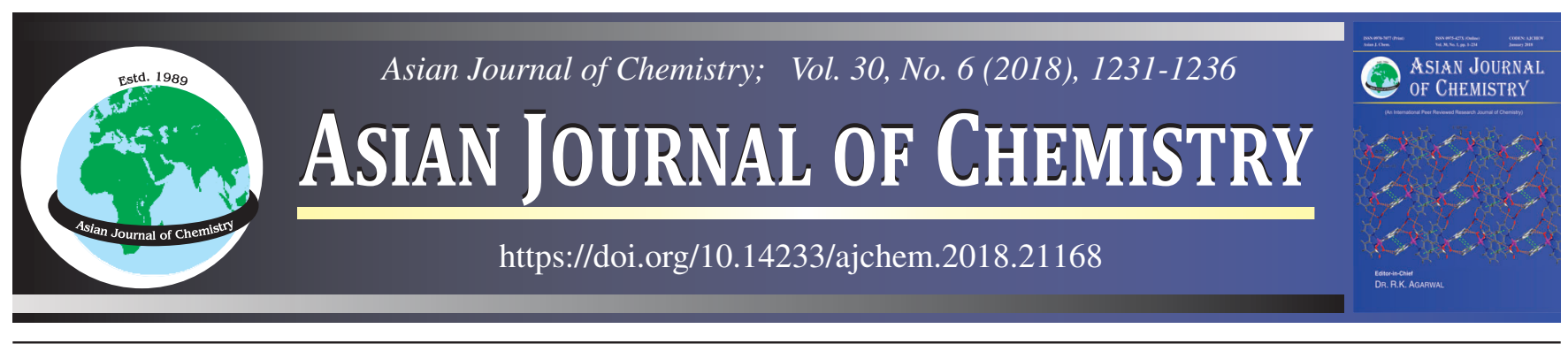

\title{
Synthesis and Antidiabetic Evaluation of 5-(2-Methoxy-6-pentadecylbenzylidene)-3-alkyl/aryl substituted thiazolidine-2,4-dione Derivatives
}

\section{UPENDAR REDDY ${ }^{1,2, *}$ and M.C. SoMASEKHARA REDDY ${ }^{3}$}

${ }^{1}$ Department of Chemistry, Rayalaseema University, Kurnool-518 007, India

${ }^{2}$ Dr. J.C.R. Bio, Plot No. 79/80, Survey No-12, Chengicherla Village, Cherlapalli Phase III Industrial Park, Hyderabad-500 039, India

${ }^{3}$ Department of Basic Sciences, G.P.R. Engineering College (Autonomous), Kurnool-518 007, India

*Corresponding author: E-mail: upendram2509@gmail.com

Received: 5 December 2017;

Accepted: 16 January 2018;

Published online: 30 April 2018;

AJC-18871

Compounds containing the thiazolidinedione moiety have been found to exhibit various pharmacological and biological activities viz., COX-2 inhibitor, antihyperglycemic, anti-inflammatory, antioxidant, cytotoxic, antimicrobial etc. The present paper describes the synthesis of 5-(2-methoxy-6-pentadecylbenzylidene)-3-alkyl/aryl-substituted thiazolidine-2,4-dione derivatives (6a-6k) in five synthetic steps utilizing anacardic acid as starting material The key steps involves (i) methylation of anacardic acid in presence of dimethyl carbonate in sealed tube (ii) methylester reduction in presence of sodium borohydride in refluxing 2-methyl-tetrahydrofuran (iii) oxidation of $1^{\circ}$ alcohol in presence of $\mathrm{CuBr}, 2$,2-bipyridine, TEMPO (iv) Condensation reaction of aldehyde with thiazolidinedione followed by alkylation. The newly synthesized 3-substituted $\mathrm{N}$-alkyl/aryl-thiazolidine derivative were evaluated for antidiabetic property (alloxan induced diabetic mice), compounds $\mathbf{6 a}, \mathbf{6 b}, \mathbf{6 c}$ showed significant decrease in fasting blood glucose (FBG) levels (when compared to standard drug with insulin).

Keywords: Anacardic acid, Dimethyl carbonate, Thiazolidine-2,4-dione, Synthesis.

L

\section{INTRODUCTION}

Diabetes mellitus is a heterogenous group of disorder, characterized by a state of chronic hyperglycemia, ensuing from a diversity of etiologies either environmental and genetics, acting jointly [1]. Characteristically, diabetes is a long term metabolic disorder with a number of complication including cardiovascular, renal, neurological, ocular and other such consistent problems [1]. Diabetes mellitus is one of the major health problems in the world today. The incidence of the disease currently is estimated to reach 300 million by the year 2025. Most cases will be of Type 2 diabetes mellitus, which sturdily connected with a sedentary life style and obesity [2]. During the last decades substituted thiazolidinediones received attention in the field of diabetes. Several thiazolidinediones serve as basic pharmacophore for various biological profiles i.e. antidiabetic, anticancer [3], antimalarial [4], aldose reductase [5] and anti-inflammatory [6], etc.

Thiazolidinedione scaffold has been recognized to play an important position in medicinal chemistry $[7,8]$. Compounds containing thiazolidinedione moiety have been found to demonstrate an extensive assortment of biological activities viz, COX-2 inhibitor [9], antihyperglycemic [10], anti-inflammatory [11], antioxidant [12], cytotoxic [13], antimicrobial [14], neuro- protective [15], antiproliferative [16], antitumor [17], MurD ligase inhibitor [18], monoamine oxidase B (MAO-B) inhibitor [19], antimalarial [20] and chemotherapeutic activities [21].

Anacardic acid (Fig. 1), a major component of natural cashew nut shell liquid (CNSL), has attracted great research interest due to its biological activities such as antitumor, antioxidant, gastro-protective and antibiotic. In addition, it has been used as a synthon for the production of a variety of biologically active compounds with increased efficiency and some of them out perform their corresponding standard material [22]. Besides the biological activities, anacardic acid has rece-ntly been found to be a potential candidate as a capping agent for the development of nanomaterials $[23,24]$.

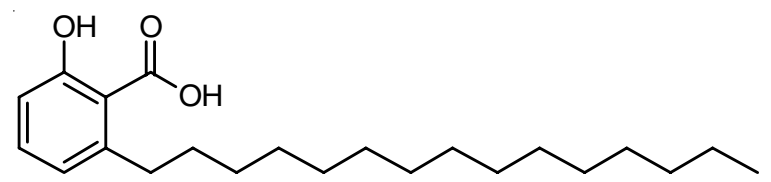

Fig. 1. Structure of anacardic acid

These observations promoted us to synthesize a new series of thiazolidinedione with higher biological activity. In the present paper, the synthesis and screening of novel thiazolidinedione derivatives embedded with anacardic acid motif for anti- 
diabetic activity was performed. The structures of the various synthesized compounds were assigned on the basis of mass, IR and ${ }^{1} \mathrm{H}$ NMR spectral data. These compounds were screened for their antidiabetic activity by using alloxan induced insulin resistance in rats.

\section{EXPERIMENTAL}

The uncorrected melting points of compounds were taken in an open capillary in a paraffin bath. All reagents used were commercial and laboratory grade, melting points were determined in open capillaries and are uncorrected. IR spectra were recorded on potassium bromide disks on a Perkin-Elmer 383 spectrophotometer. ${ }^{1} \mathrm{H}$ NMR spectra were obtained on Varian $400 \mathrm{MHz}$ instrument and Varian $400 \mathrm{MHz}$, with TMS as internal Standard and chemical shifts are expressed in $\delta \mathrm{ppm}$ solvent used in $\mathrm{CDCl}_{3}$ and mass spectrum on a Hewelett Packard mass spectrometer operating at $70 \mathrm{ev}$, purity of the compounds were checked by TLC, which is performed with E. Merck pre coated silica gel plates (60 F-254) with iodine as a developing agent. Acme, India silica gel, 60-120 mesh for column chromatography is used.

Preparation of methyl (2-methoxy-6-pentadecyl)benzoate (2): In a sealed tube vessel, was added 2-hydroxy-6-pentadecylbenzoic acid (1, $10 \mathrm{~g}, 28.70 \mathrm{mmol})$, 2-methyl tetrahydrofuran $(60 \mathrm{~mL})$, dimethylcarbonate $(5.17 \mathrm{~g}, 57.40 \mathrm{mmol})$ followed by DBU $(0.44 \mathrm{~g}, 2.90 \mathrm{mmol})$ and the cap was screwed tightly. The reaction mixture was heated to $140{ }^{\circ} \mathrm{C}$ for $17 \mathrm{~h}$. After cooling, the reaction contents was diluted with cyclopentyl methyl ether $(25 \mathrm{~mL})$, washed with $10 \%$ solution of sodium bicarbonate solution $(3 \times 15 \mathrm{~mL})$, washed with $1 \mathrm{M} \mathrm{HCl}(3 \times 25$ $\mathrm{mL})$ and with water $(3 \times 25 \mathrm{~mL})$, dried over sodium sulphate, filtered and concentrated to afford methyl 2-methoxy-6pentadecylbenzoate (3). Pale yellow solid; Yield: 9.0 g, $84 \%$; m.p.: 36-37 ${ }^{\circ}$; IR (KBr, $v_{\max }, \mathrm{cm}^{-1}$ ): 3004 (Ar-CH str, 2921 (-CH str), 1732 (-C=O str), 1460 (-C=C-, str), 1105 (-C-OC-, str); ${ }^{1} \mathrm{H}$ NMR (400 MHz, $\left.\mathrm{CDCl}_{3}\right) \delta: 7.25(\mathrm{t}, J=8.0 \mathrm{~Hz}, 1 \mathrm{H})$, $6.82(\mathrm{~d}, J=8.0 \mathrm{~Hz}, 1 \mathrm{H}), 6.75(\mathrm{~d}, J=8.4 \mathrm{~Hz}, 1 \mathrm{H}), 3.90(\mathrm{~s}$, $3 \mathrm{H}), 3.81(\mathrm{~s}, 3 \mathrm{H}), 2.53(\mathrm{t}, J=8.0 \mathrm{~Hz}, 2 \mathrm{H}), 1.53-1.60(\mathrm{~m}, 2 \mathrm{H})$, 1.25 (brs, 24H), 0.88 (t, $J=6.8 \mathrm{~Hz}, 3 \mathrm{H}$ ); ESI-MS: $m / z, 377$ $[\mathrm{M}+\mathrm{H}]^{+}$.

Preparation of (2-methoxy-6-pentadecylphenyl)methanol (3): To a stirred suspension of methyl 2-methoxy6-pentadecylbenzoate $(2,5.0 \mathrm{~g}, 13.28 \mathrm{mmol})$ in 2-methyl THF (75 mL) was added sodium borohydride ( $2 \mathrm{~g}, 52.86 \mathrm{mmol})$ and heated under reflux for $1 \mathrm{~h}$. The above reaction mixture was diluted with methanol $(75 \mathrm{~mL})$ and was further refluxed for $1 \mathrm{~h}$. The reaction mixture was cooled to room temperature and quenched with a saturated solution of $\mathrm{NH}_{4} \mathrm{Cl}(50 \mathrm{~mL})$ for $2 \mathrm{~h}$ and diluted with isopropylacetate. The organic extracts was washed with water followed by brine solution, dried over $\mathrm{Na}_{2} \mathrm{SO}_{4}$ and concentrated under reduced pressure to obtain (2-methoxy-6-pentadecylphenyl)methanol (3). Off white solid; Yield: 3 g, $81 \%$; m.p.: 60-62 ${ }^{\circ} \mathrm{C}$; IR (KBr, $\left.v_{\max }, \mathrm{cm}^{-1}\right): 3367$ ($\mathrm{OH}$ str), 3004 (Ar-CH,str), 2853 (-CH str), 1457 (-C=C, str), 1080 (-C-O-C, str); ${ }^{1} \mathrm{H}$ NMR (400 MHz, $\mathrm{CDCl}_{3}$ ) $\delta: 7.20$ (t, J $=8.0 \mathrm{~Hz}, 1 \mathrm{H}), 6.82(\mathrm{~d}, J=7.6 \mathrm{~Hz}, 1 \mathrm{H}), 6.77(\mathrm{~d}, J=8.0 \mathrm{~Hz}$, $1 \mathrm{H}), 4.75(\mathrm{~d}, J=6.4 \mathrm{~Hz}, 2 \mathrm{H}), 3.87(\mathrm{~s}, 3 \mathrm{H}), 2.68(\mathrm{t}, J=6.4 \mathrm{~Hz}$, $2 \mathrm{H}), 2.37(\mathrm{t}, J=6.4 \mathrm{~Hz}, 1 \mathrm{H}), 1.53-1.58$ (m, 2H), 1.27 (brs,
24H), 0.89 (t, $J=7.2 \mathrm{~Hz}, 3 \mathrm{H}, \mathrm{D}_{2} \mathrm{O}$ exchangeable $\left.\mathrm{OH}\right)$; ESIMS: $\mathrm{m} / z, 349[\mathrm{M}+\mathrm{H}]^{+}$.

Preparation of 2-methoxy-6-pentadecylbenzaldehyde (4): To a stirred solution of 2-methoxy-6-pentadecylphenyl)methanol $(3,2 \mathrm{~g}, 5.74 \mathrm{mmol})$ in acetone $(30 \mathrm{~mL})$ was added sequentially $\mathrm{CuBr}$ (10 mol \%), bpy (2,2'-bipyridine, $10 \mathrm{~mol}$ $\%)$ and TEMPO (10 mol \%) while the solution was stirred for 15 min and then $\mathrm{N}$-methyl imidazole (10 mol \%) was added. The reaction mixture was stirred rapidly while open to the air for $2 \mathrm{~h}$ (until the reaction is complete). The reaction mixture was diluted with water $(50 \mathrm{~mL})$ and extracted with pentane $(3 \times 30 \mathrm{~mL})$, the organic layer was separated and dried over anhydrous $\mathrm{Na}_{2} \mathrm{SO}_{4}$, filtered and evaporated to afford 2-methoxy6-pentadecylbenzaldehyde (4). Pale yellow solid; Yield: $1.4 \mathrm{~g}$ (71\%); m.p.: 70-72 ${ }^{\circ} \mathrm{C}$; IR (KBr, $\left.v_{\max }, \mathrm{cm}^{-1}\right)$ : 1687 (C=O stretching), 1589 (C-O stretching); ${ }^{1} \mathrm{H} \mathrm{NMR}\left(\mathrm{CDCl}_{3}, 400 \mathrm{MHz}\right)$ : $\delta, 10.62(\mathrm{~s}, 1 \mathrm{H}), 7.39(\mathrm{t}, J=7.6 \mathrm{~Hz}, 1 \mathrm{H}), 6.80(\mathrm{~d}, J=8.4 \mathrm{~Hz}$, $2 \mathrm{H}), 3.88$ (s, 3H), $2.92(\mathrm{t}, J=8.0 \mathrm{~Hz}, 2 \mathrm{H}), 1.51-1.57(\mathrm{~m}, 2 \mathrm{H})$, 1.25 (brs, 24H), 0.88 (t, $J=6.8 \mathrm{~Hz}, 3 \mathrm{H}$ ); ESI-MS: $m / z, 347.29$ $[\mathrm{M}+\mathrm{H}]^{+}$.

Preparation of $(E)$-5-(2-methoxy-6-pentadecylbenzylidene)thiazolidine-2,4-dione (5): To a solution of compound $4(1.0 \mathrm{~g}, 2.890 \mathrm{mmol})$ in toluene $(15 \mathrm{~mL})$ was added thiazolidinedione (339 mg, $2.89 \mathrm{mmol}$ ), ammonium acetate (669 mg, $8.670 \mathrm{mmol})$ and acetic acid $(1 \mathrm{~mL})$ at room temperature. Reaction mixture was refluxed for $3 \mathrm{~h}$, reaction mixture was distilled off crude compound was re-dissolved in ethyl acetate $(50 \mathrm{~mL})$ with water $(50 \mathrm{~mL})$, brine solution $(20 \mathrm{~mL})$ dried over anhydrous $\mathrm{Na}_{2} \mathrm{SO}_{4}$, filtered and evaporated under vacuum to obtain 5-(2-methoxy-6-pentadecyl-benzylidene)thiazolidine2,4-dione (5). Cream colour solid; Yield: $650 \mathrm{mg},(50.4 \%)$; m.p.: 82-83 ${ }^{\circ} \mathrm{C}$; IR (KBr pellet, $\mathrm{cm}^{-1}$ ): 3184 (Ar-CH, str), 2912 (-CH str), 1739, 1699 (C=O str, thiazolidine carbonyl), 1597 (-C=C- str), 1078 (-C-O-C str), 763 (C-SC- str); ${ }^{1} \mathrm{H}$ NMR $\left(\mathrm{CDCl}_{3}, 400 \mathrm{MHz}\right): \delta 7.92(\mathrm{~s}, 1 \mathrm{H}), 7.22(\mathrm{dd}, J=7.6,16 \mathrm{~Hz}$, $1 \mathrm{H}), 6.80(\mathrm{~d}, J=7.6 \mathrm{~Hz}, 1 \mathrm{H}), 6.70(\mathrm{~d}, J=7.6 \mathrm{~Hz}, 1 \mathrm{H}), 5.66(\mathrm{~s}$, 2H), 3.80 (s, 3H), 2.58 (t, $J=7.6 \mathrm{~Hz}, 2 \mathrm{H}), 1.48-1.44(\mathrm{~m}, 2 \mathrm{H})$, 1.21-1.17 (m, 24H), $0.80(\mathrm{t}, J=7.6 \mathrm{~Hz}, 3 \mathrm{H}) ;{ }^{13} \mathrm{C}$ NMR $(100$ $\left.\mathrm{MHz}, \mathrm{CDCl}_{3}\right), \delta: 167.5,165.8,155.8,143.7,130.2,130.2,125.8$, 121.1, 119.3, 107.5, 54.0, 32.7, 30.9, 30.0, 29.0, 28.6(6C), 28.5, 28.4, 28.3 (2C), 21.6, 13.0; MS (ESI) $\mathrm{m} / \mathrm{z}: 446.1[\mathrm{M}+\mathrm{H}]^{+}$.

General procedure for the N-alkylation of $(E)-5-(2-$ methoxy-6-pentadecylbenzylidene)thiazolidine-2,4-dione (5) to produce corresponding $\mathrm{N}$-alkyl/aryl substitutedthiazolidine-2,4-dione derivatives: To a stirred solution of compound $\mathbf{5}$ (100 mg, $0.225 \mathrm{mmol}$ ) in DMF (1 mL) was added potassium carbonate $(0.27 \mathrm{mmol})$ followed by the corresponding alkyl bromides $(0.225 \mathrm{mmol})$, benzyl bromide $(0.225$ $\mathrm{mmol})$ and phenacyl bromide $(0.225 \mathrm{mmol})$ and heated to $80^{\circ} \mathrm{C}$ for 1-3 h. After the completion of the reaction (monitored by TLC), the reaction mixture was diluted with water and extracted with ethylacetate $(2 \times 5 \mathrm{~mL})$. The organic layer was washed with water $(3 \times 5 \mathrm{~mL})$ followed by brine solution, separated and dried over $\mathrm{Na}_{2} \mathrm{SO}_{4}$, filtered and evaporated under reduced pressure to obtain the corresponding 2,4-thiazolidine derivatives 6a-6k.

(E)-5-(2-methoxy-6-pentadecylbenzylidene)-3-ethylthiazolidine-2,4-dione (6a): Pale yellow viscous liquid; Yield: 
$88 \% ;{ }^{1} \mathrm{H} \mathrm{NMR}\left(\mathrm{CDCl}_{3}, 400 \mathrm{MHz}\right): \delta 8.0(\mathrm{~s}, 1 \mathrm{H}), 7.30(\mathrm{dd}$, $J=7.6,10.8 \mathrm{~Hz}, 1 \mathrm{H}), 6.90(\mathrm{~d}, J=7.6 \mathrm{~Hz}, 1 \mathrm{H}), 6.78(\mathrm{~d}, J=$ $7.6 \mathrm{~Hz}, 1 \mathrm{H}), 3.84(\mathrm{~s}, 2 \mathrm{H}), 3.80(\mathrm{q}, J=6.8 \mathrm{~Hz}, 2 \mathrm{H}), 2.62(\mathrm{t}, J$ $=7.6 \mathrm{~Hz}, 2 \mathrm{H}), 1.58-1.52(\mathrm{~m}, 2 \mathrm{H}), 1.38-1.22(\mathrm{~m}, 27 \mathrm{H}), 0.90$ (t, $J=7.6 \mathrm{~Hz}, 3 \mathrm{H})$; MS (ESI) $\mathrm{m} / \mathrm{z}: 474.1(\mathrm{M}-\mathrm{H})^{+}$.

(E)-5-(2-methoxy-6-pentadecylbenzylidene)-3-propylthiazolidine-2,4-dione (6b): Pale yellow viscous liquid; Yield: $85 \%$; IR (KBr pellet, $\left.\mathrm{cm}^{-1}\right)$ : 2916 (-CH str), 1732 (-C=O, ethylester carbonyl), 1691,1604 (C=O str, thiazolidine carbonyl), 1593 (-C=C-str), 1026 (-C-O-C str), 800 (C-S-C str); ${ }^{1} \mathrm{H}$ NMR $\left(\mathrm{CDCl}_{3}, 400 \mathrm{MHz}\right): \delta 8.0(\mathrm{~s}, 1 \mathrm{H}), 7.30(\mathrm{dd}, J=7.6,10.8 \mathrm{~Hz}$, $1 \mathrm{H}), 6.90(\mathrm{~d}, J=7.6 \mathrm{~Hz}, 1 \mathrm{H}), 6.80(\mathrm{~d}, J=7.6 \mathrm{~Hz}, 1 \mathrm{H}), 3.82$ (s, 2H), 3.75 (q, $J=6.8 \mathrm{~Hz}, 2 \mathrm{H}), 2.62(\mathrm{t}, J=7.6 \mathrm{~Hz}, 2 \mathrm{H})$, $1.58-1.52(\mathrm{~m}, 2 \mathrm{H}), 1.38-1.22(\mathrm{~m}, 24 \mathrm{H}), 0.98(\mathrm{t}, J=6.8 \mathrm{~Hz}$, $3 \mathrm{H}), 0.92(\mathrm{t}, J=6.8 \mathrm{~Hz}, 3 \mathrm{H})$; ESI-MS: $m / z, 488.1[\mathrm{M}-\mathrm{H}]^{+}$.

Ethyl 2-[(E)-5-(2-methoxy-6-pentadecylbenzylidene)2,4-dioxothiazolidin-3-yl]acetate (6c): Pale yellow solid; Yield: $78 \%$; m.p.: 50-51 ${ }^{\circ} \mathrm{C}$; IR (KBr pellet, $\mathrm{cm}^{-1}$ ): $2918(-\mathrm{CH}$ str), 1732 (-C=O str, ethylester group), 1691, 1604 (-C=O, thiazolidine group), 1593 (-C=C- str), 1026 (-C-O-C str), 800 (C-S-C str); ${ }^{1} \mathrm{H}$ NMR $\left(\mathrm{CDCl}_{3}, 400 \mathrm{MHz}\right): \delta 7.98(\mathrm{~s}, 1 \mathrm{H}), 7.22$ $(\mathrm{dd}, J=8.0,16 \mathrm{~Hz}, 1 \mathrm{H}), 6.80(\mathrm{~d}, J=7.6 \mathrm{~Hz}, 1 \mathrm{H}), 6.70(\mathrm{~d}, J=$ $7.6 \mathrm{~Hz}, 1 \mathrm{H}), 4.38$ (s, 2H), 4.18 (q, J = 7.2 Hz, 2H), $3.80(\mathrm{~s}, 3 \mathrm{H})$, 2.58 (t, $J=7.6 \mathrm{~Hz}, 2 \mathrm{H}), 1.48-1.44(\mathrm{~m}, 2 \mathrm{H}), 1.21-1.17(\mathrm{~m}$, $24 \mathrm{H}), 0.80$ (t, $J=7.6 \mathrm{~Hz}, 3 \mathrm{H})$; ESI-MS: $m / z, 532.5[\mathrm{M}+\mathrm{H}]^{+}$.

(E)-5-(2-Methoxy-6-pentadecylbenzylidene)-3-benzylthiazolidine-2,4-dione (6d): Colourless viscous liquid; Yield: $82 \%$; ${ }^{1} \mathrm{H}$ NMR $\left(\mathrm{CDCl}_{3}, 400 \mathrm{MHz}\right): \delta{ }^{1} \mathrm{H} \mathrm{NMR}\left(\mathrm{CDCl}_{3}, 400\right.$ MHz): $\delta 7.98(\mathrm{~d}, J=8.0 \mathrm{~Hz}, 1 \mathrm{H}), 7.34-7.28(\mathrm{~m}, 5 \mathrm{H}), 7.20$ (dd, $J=7.8,10.4 \mathrm{~Hz}, 1 \mathrm{H}), 6.76(\mathrm{~d}, J=7.8 \mathrm{~Hz}, 1 \mathrm{H}), 6.68(\mathrm{~d}$, $J=7.8 \mathrm{~Hz}, 1 \mathrm{H}), 4.80(\mathrm{~s}, 2 \mathrm{H}), 3.82(\mathrm{~s}, 3 \mathrm{H}), 2.62(\mathrm{t}, J=7.6 \mathrm{~Hz}$, $2 \mathrm{H}), 1.44(\mathrm{t}, J=7.6 \mathrm{~Hz}, 2 \mathrm{H}), 1.28-1.20(\mathrm{~m}, 24 \mathrm{H}), 0.80(\mathrm{t}, J=$ $7.6 \mathrm{~Hz}, 3 \mathrm{H})$; ESI-MS: $m / z, 554.1[\mathrm{M}+\mathrm{H}]^{+}$.

2-[\{(E)-5-(2-Methoxy-6-pentadecylbenzylidene)-2,4dioxothiazolidin-3-yl\}methyl]benzonitrile (6e): Off white solid; Yield: $86 \%$; m.p.: $92-93{ }^{\circ} \mathrm{C} ;{ }^{1} \mathrm{H} \mathrm{NMR}\left(\mathrm{CDCl}_{3}, 400 \mathrm{MHz}\right)$ : $\delta{ }^{1} \mathrm{H}$ NMR $\left(\mathrm{CDCl}_{3}, 400 \mathrm{MHz}\right): \delta 8.0(\mathrm{~s}, 1 \mathrm{H}), 7.90(\mathrm{~d}, J=8.0$ $\mathrm{Hz}, 1 \mathrm{H}), 7.70(\mathrm{t}, J=8.2 \mathrm{~Hz}, 1 \mathrm{H}), 7.50(\mathrm{t}, J=8.2 \mathrm{~Hz}, 1 \mathrm{H}), 7.40$ $(\mathrm{d}, J=8.0 \mathrm{~Hz}, 1 \mathrm{H}), 7.36(\mathrm{t}, J=7.4 \mathrm{~Hz}, 1 \mathrm{H}), 7.0(\mathrm{~d}, J=7.6 \mathrm{~Hz}$, $1 \mathrm{H}), 6.90(\mathrm{~d}, J=7.6 \mathrm{~Hz}, 1 \mathrm{H}), 5.0(\mathrm{~s}, 2 \mathrm{H}), 3.82(\mathrm{~s}, 3 \mathrm{H}), 2.62(\mathrm{t}$, $J=7.6 \mathrm{~Hz}, 2 \mathrm{H}), 1.52-1.48(\mathrm{~m}, 2 \mathrm{H}), 1.35-1.12(\mathrm{~m}, 24 \mathrm{H}), 0.92$ (t, $J=7.6 \mathrm{~Hz}, 3 \mathrm{H})$; ESI-MS: $m / z, 561.2[\mathrm{M}+\mathrm{H}]^{+}$.

3-[\{(E)-5-(2-Methoxy-6-pentadecylbenzylidene)-2,4dioxothiazolidin-3-yl\}methyl]benzonitrile (6f): Off white solid; Yield: $82 \%$; m.p.: $90-91{ }^{\circ} \mathrm{C} ;{ }^{1} \mathrm{H} N M R\left(\mathrm{CDCl}_{3}, 400 \mathrm{MHz}\right)$ : $\delta 7.96(\mathrm{~d}, J=8.0 \mathrm{~Hz}, 1 \mathrm{H}), 7.40-7.36(\mathrm{~m}, 4 \mathrm{H}), 7.26(\mathrm{dd}, J=$ $7.8,10.2 \mathrm{~Hz}, 1 \mathrm{H}), 6.84(\mathrm{~d}, J=7.8 \mathrm{~Hz}, 1 \mathrm{H}), 6.76(\mathrm{~d}, J=7.8$ $\mathrm{Hz}, 1 \mathrm{H}), 4.84(\mathrm{~s}, 2 \mathrm{H}), 3.86(\mathrm{~s}, 3 \mathrm{H}), 2.66(\mathrm{t}, J=7.6 \mathrm{~Hz}, 2 \mathrm{H})$, $1.46(\mathrm{t}, J=7.6 \mathrm{~Hz}, 2 \mathrm{H}), 1.26-1.20(\mathrm{~m}, 24 \mathrm{H}), 0.82(\mathrm{t}, J=7.6$ $\mathrm{Hz}, 3 \mathrm{H})$; ESI-MS: $m / z, 561.3[\mathrm{M}+\mathrm{H}]^{+}$.

(E)-5-(2-Methoxy-6-pentadecylbenzylidene)-3-(3chlorobenzyl)thiazolidine-2,4-dione (6g): Off white solid; Yield: $82 \%$; m.p.: $101-102{ }^{\circ} \mathrm{C}$; ${ }^{1} \mathrm{H} \mathrm{NMR}\left(\mathrm{CDCl}_{3}, 400 \mathrm{MHz}\right)$ : $\delta 7.98(\mathrm{~d}, J=8.0 \mathrm{~Hz}, 1 \mathrm{H}), 7.38-7.34(\mathrm{~m}, 4 \mathrm{H}), 7.24(\mathrm{dd}, J=$ $7.8,11.0 \mathrm{~Hz}, 1 \mathrm{H}), 6.80(\mathrm{~d}, J=7.8 \mathrm{~Hz}, 1 \mathrm{H}), 6.70(\mathrm{~d}, J=7.8$ $\mathrm{Hz}, 1 \mathrm{H}), 4.80(\mathrm{~s}, 2 \mathrm{H}), 3.82(\mathrm{~s}, 3 \mathrm{H}), 2.62(\mathrm{t}, J=7.6 \mathrm{~Hz}, 2 \mathrm{H})$, $1.44(\mathrm{t}, J=7.6 \mathrm{~Hz}, 2 \mathrm{H}), 1.24-1.18(\mathrm{~m}, 24 \mathrm{H}), 0.80$ (t, $J=7.6$ Hz, 3H); ESI-MS: $m / z, 570.1[\mathrm{M}+\mathrm{H}]^{+}$.
(E)-5-(2-methoxy-6-pentadecylbenzylidene)-3-(4chlorobenzyl)thiazolidine-2,4-dione (6h): Yellow oily liquid; Yield: $86 \%$; ${ }^{1} \mathrm{H}$ NMR $\left(\mathrm{CDCl}_{3}, 400 \mathrm{MHz}\right): \delta 7.98(\mathrm{~s}, 1 \mathrm{H}), 7.90$ $(\mathrm{d}, J=7.2 \mathrm{~Hz}, 2 \mathrm{H}), 7.56(\mathrm{~d}, J=7.2 \mathrm{~Hz}, 2 \mathrm{H}), 7.42(\mathrm{t}, J=7.8$ $\mathrm{Hz}, 1 \mathrm{H}), 7.04(\mathrm{~d}, J=7.8 \mathrm{~Hz}, 1 \mathrm{H}), 6.92(\mathrm{~d}, J=7.8 \mathrm{~Hz}, 1 \mathrm{H})$, $4.88(\mathrm{~s}, 2 \mathrm{H}), 3.80(\mathrm{~s}, 3 \mathrm{H}), 2.62(\mathrm{t}, J=6.8 \mathrm{~Hz}, 2 \mathrm{H}), 1.48-1.42$ (m, 2H), 1.32-1.26 (m, 24H), 0.90 (t, $J=7.6 \mathrm{~Hz}, 3 \mathrm{H})$; ESIMS: $m / z, 570.3[\mathrm{M}+\mathrm{H}]^{+}$.

4-[\{(E)-5-(2-methoxy-6-pentadecylbenzylidene)-2,4dioxothiazolidin-3-yl\}methyl]benzonitrile (6i): Pale yellow solid; Yield: $84 \%$; m.p.: $118-119{ }^{\circ} \mathrm{C} ;{ }^{1} \mathrm{H}$ NMR $\left(\mathrm{CDCl}_{3}, 400\right.$ MHz): $\delta 8.01(\mathrm{~s}, 1 \mathrm{H}), 7.92(\mathrm{~d}, J=7.2 \mathrm{~Hz}, 2 \mathrm{H}), 7.52(\mathrm{~d}, J=$ $7.2 \mathrm{~Hz}, 2 \mathrm{H}), 7.38(\mathrm{t}, J=7.8 \mathrm{~Hz}, 1 \mathrm{H}), 7.0(\mathrm{~d}, J=7.8 \mathrm{~Hz}, 1 \mathrm{H})$, $6.90(\mathrm{~d}, J=7.8 \mathrm{~Hz}, 1 \mathrm{H}), 4.90(\mathrm{~s}, 2 \mathrm{H}), 3.82(\mathrm{~s}, 3 \mathrm{H}), 2.60(\mathrm{t}, J$ $=6.8 \mathrm{~Hz}, 2 \mathrm{H}), 1.50-1.42(\mathrm{~m}, 2 \mathrm{H}), 1.30-1.26(\mathrm{~m}, 24 \mathrm{H}), 0.90$ (t, $J=7.6 \mathrm{~Hz}, 3 \mathrm{H})$; ESI-MS: $m / z, 561.0[\mathrm{M}+\mathrm{H}]^{+}$.

(E)-5-(2-Hexadecyl-6-methoxy-benzylidene)-3-(2-oxo2-phenyl-ethyl)-thiazolidine-2,4-dione (6j): Colourless viscous liquid; Yield: $78 \%$; ${ }^{1} \mathrm{H}$ NMR $\left(\mathrm{CDCl}_{3}, 400 \mathrm{MHz}\right): \delta$ 8.06-7.78 $(\mathrm{m}, 5 \mathrm{H}), 7.96(\mathrm{~s}, 1 \mathrm{H}), 7.40(\mathrm{t}, J=7.6,1 \mathrm{H}), 6.92(\mathrm{~d}, J=7.6$ $\mathrm{Hz}, 1 \mathrm{H}), 6.86(\mathrm{~d}, J=7.6 \mathrm{~Hz}, 1 \mathrm{H}), 5.26(\mathrm{~s}, 2 \mathrm{H}), 3.80(\mathrm{~s}, 3 \mathrm{H})$, 2.60 (t, $J=7.6 \mathrm{~Hz}, 2 \mathrm{H}), 1.50$ (t, $J=7.8 \mathrm{~Hz}, 2 \mathrm{H}), 1.20-1.14$ $(\mathrm{m}, 24 \mathrm{H}), 0.88(\mathrm{t}, J=7.6 \mathrm{~Hz}, 3 \mathrm{H})$; ESI-MS: $\mathrm{m} / z, 564.1[\mathrm{M}+\mathrm{H}]^{+}$.

(E)-3-[2-(4-Bromo-phenyl)-2-oxo-ethyl]-5-(2-hexadecyl6-methoxy-benzylidene)thiazolidine-2,4-dione (6k): Pale yellow visous liquid; Yield: $80 \%$; ${ }^{1} \mathrm{H} \mathrm{NMR}\left(\mathrm{CDCl}_{3}, 400 \mathrm{MHz}\right)$ : $\delta 8.06(\mathrm{~d}, J=8.2 \mathrm{~Hz}, 2 \mathrm{H}), 8.0(\mathrm{~s}, 1 \mathrm{H}), 7.80(\mathrm{~d}, J=8.2 \mathrm{~Hz}$, $2 \mathrm{H}), 7.40(\mathrm{t}, J=7.6,1 \mathrm{H}), 7.00(\mathrm{~d}, J=7.6 \mathrm{~Hz}, 1 \mathrm{H}), 6.90(\mathrm{~d}$, $J=7.6 \mathrm{~Hz}, 1 \mathrm{H}), 5.30(\mathrm{~s}, 2 \mathrm{H}), 3.84(\mathrm{~s}, 3 \mathrm{H}), 2.64(\mathrm{t}, J=7.6 \mathrm{~Hz}$, $2 \mathrm{H}), 1.52(\mathrm{t}, J=7.8 \mathrm{~Hz}, 2 \mathrm{H}), 1.22-1.18(\mathrm{~m}, 24 \mathrm{H}), 0.82(\mathrm{t}, J=$ $7.6 \mathrm{~Hz}, 3 \mathrm{H})$; ESI-MS: $\mathrm{m} / \mathrm{z}, 642.1[\mathrm{M}+\mathrm{H}]^{+}$.

Pharmacological experimental section $[25,26]$

Experimental animals: Swiss albino mice weighing 25$30 \mathrm{~g}$ were used for this study. Animals were grouped and housed for one week to acclimatize to laboratory conditions before starting the experiment. Animals were fed with standard diet and water ad libitum and standard laboratory conditions. $12 \mathrm{~h}$ prior to an experiment; the animals were deprived of food but not water.All the experimental procedures were carried out accordance with CPCSEA guidelines. The Institutional Animal Ethics Committee was approved the experimental protocol.

Induction of diabetes: To the overnight-fasted Swiss albino mice was injected with alloxan monohydrate $(150 \mathrm{mg} /$ $\mathrm{kg}$ ) in saline buffer administer through intraperitoneally. In order to prevent hypoglycaemia after injection of alloxan, animals were fed with $20 \%$ w/v glucose solution. After $72 \mathrm{~h}$ the fasting blood glucose levels (FBG) were determined by using ONETOUCH select simple (J\&J) Glucometer strips (diabetic control, Table-1). Mice with $<250 \mathrm{mg} / \mathrm{dl}$ were excluded from experiment and mice with $>250 \mathrm{mg} / \mathrm{dl}$ are selected to diabetic study and divide them into groups.

Protocol for animal: Animals were separated into 13 sets of 6 mice $(\mathrm{n}=6)$ : (i) diabetic animals (vehicle) labeled as set1 , received $1 \mathrm{~mL}$ of CMC ( $0.5 \%)$; (ii) diabetic animals labeled as set-2, received insulin as a standard $2 \mathrm{IU} / \mathrm{kg} \mathrm{SC.50} \mathrm{mg/Kg}$. (iii) Set (3-13) diabetic animals received compounds 6a-k in a single dose $(10 \mathrm{mg} / \mathrm{kg}$ body weight per oral) respectively for seven days incessantly. 
TABLE-1

FBG LEVELS OF THIAZOLIDINE DERIVATIVES (6a-k)

\begin{tabular}{ccccc}
\hline Groups & After $1^{\text {st }}$ day of treatment & After $3^{\text {rd }}$ day of treatment & After $7^{\text {th }}$ day of treatment & Hyperglycemic activity $(\%)$ \\
\hline Control & $93 \pm 1.29$ & $92.5 \pm 1.84$ & $95.5 \pm 2.02$ & - \\
Insulin & $321.8 \pm 4.76^{* * *}$ & $108.8 \pm 3.497 * * *$ & $108.3 \pm 2.689 * * *$ & 66.34 \\
6a & $304.3 \pm 3.65 \mathrm{~ns}$ & $222.0 \pm 2.45^{*}$ & $120.0 \pm 1.10^{* * *}$ & 60.52 \\
6b & $288.0 \pm 3.12^{* *}$ & $210.3 \pm 1.11^{* * *}$ & $115.3 \pm 1.931^{* * *}$ & 60.06 \\
6c & $286.5 \pm 5.31^{*}$ & $187.8 \pm 4.32^{* * *}$ & $113.0 \pm 3.440^{* * *}$ & 60.55 \\
6d & $303.3 \pm 6.03^{*}$ & $224.0 \pm 6.41^{* * *}$ & $136.0 \pm 4.54 * * *$ & 55.15 \\
6e & $317.3 \pm 5.16 \mathrm{~ns}$ & $288.0 \pm 4.52^{*}$ & $226.5 \pm 2.781^{* * *}$ & 28.61 \\
6f & $310.3 \pm 3.10^{* *}$ & $242.0 \pm 5.54^{*}$ & $214.5 \pm 1.454^{* * *}$ & 30.07 \\
$6 \mathrm{~g}$ & $292.8 \pm 4.0 \mathrm{~ns}$ & $222.3 \pm 3.11^{* * *}$ & $147.3 \pm 1.931^{* * *}$ & 49.69 \\
6h & $284.3 \pm 3.11^{* *}$ & $247.0 \pm 2.58^{* * *}$ & $157.5 \pm 3.663^{* * *}$ & 44.60 \\
$6 \mathrm{i}$ & $310.3 \pm 6.86 \mathrm{~ns}$ & $293.0 \pm 6.02^{*}$ & $208.5 \pm 5.951^{* * *}$ & 32.80 \\
$6 \mathrm{j}$ & $315.3 \pm 4.95 \mathrm{~ns}$ & $214.0 \pm 3.62^{* * *}$ & $133.0 \pm 3.16^{* * *}$ & 57.80 \\
$6 \mathrm{k}$ & $280.5 \pm 2.11 * *$ & $194.8 \pm 4.32^{* * *}$ & $118.0 \pm 2.520^{* * *}$ & 57.93 \\
\hline
\end{tabular}

Data are expressed as mean \pm SEM $(\mathrm{n}=4)$ and analyzed by one way ANOVA followed by Tukey's multiple comparison test. $* * * \mathrm{P}<0.001, * * \mathrm{P}<$ $0.01,{ }^{*} \mathrm{P}<0.05$ as compared to the diabetic control group.

Measurement of blood glucose levels: Each individual reading was noted by withdrawing blood sample from the tail vain. Measurement of blood glucose was taken at intervals of $0,3^{\text {rd }}$ and $7^{\text {th }}$ day. By snipping the tip of the tail, blood samples were withdrawn from a tail vein and the blood glucose level was measured at the end of 0, $3^{\text {rd }}$ and $7^{\text {th }}$ day (by Accu Sure Blood Glucose Monitoring System, Dr. Gene Health \& Wellness.

\section{RESULTS AND DISCUSSION}

The synthesis of 5-(2-methoxy-6-pentadecylbenzylidene)3-alkyl/aryl-substituted thiazolidine-2,4-dione derivatives (6a-6k) is illustrated in Scheme-I. These derivatives were prepared in five steps utilizing anacardic acid as starting material [27]. Methylation of anacardic acid (1) was achieved in presence of dimethyl carbonate [28], 1,8-diazabicyclo[5.4.0] undec-7-ene (DBU) in 2-Me THF in sealed tube at $140^{\circ} \mathrm{C}$ for $17 \mathrm{~h}$ produced methyl (2-methoxy-6-pentadecyl)benzoate (2) in $84 \%$ yield. Sodium borohydride reduction of 2-methoxy-6-pentadecylbenzoate (2) in presence of 2-Me THF at reflux for $1 \mathrm{~h}$ yielded (2-methoxy-6-pentadecylphenyl)methanol (3) in $81 \%$. The oxidation of alcohol 3 [29] was carried out in presence of $\mathrm{CuBr}$, 2,2-bipyridine, TEMPO, $\mathrm{N}$-methyl imidazole in acetone at room temperature resulted in the formation of 2-methoxy-6pentadecylbenzaldehyde 4 in $71 \%$ yield. Condensation of

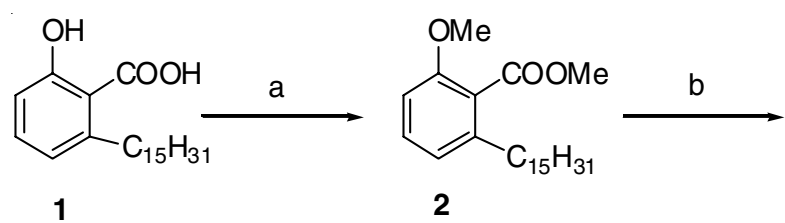

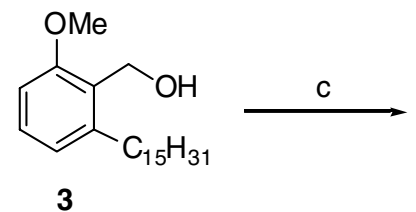<smiles>COc1cccc(C(C)(C)C)c1/C=C1\SC(=O)NC1=O</smiles>

5<smiles>[R]N1C(=O)S/C(=C\c2c(C)cccc2OC)C1=O</smiles>

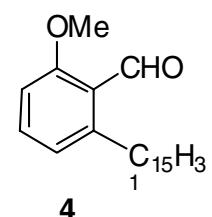

4

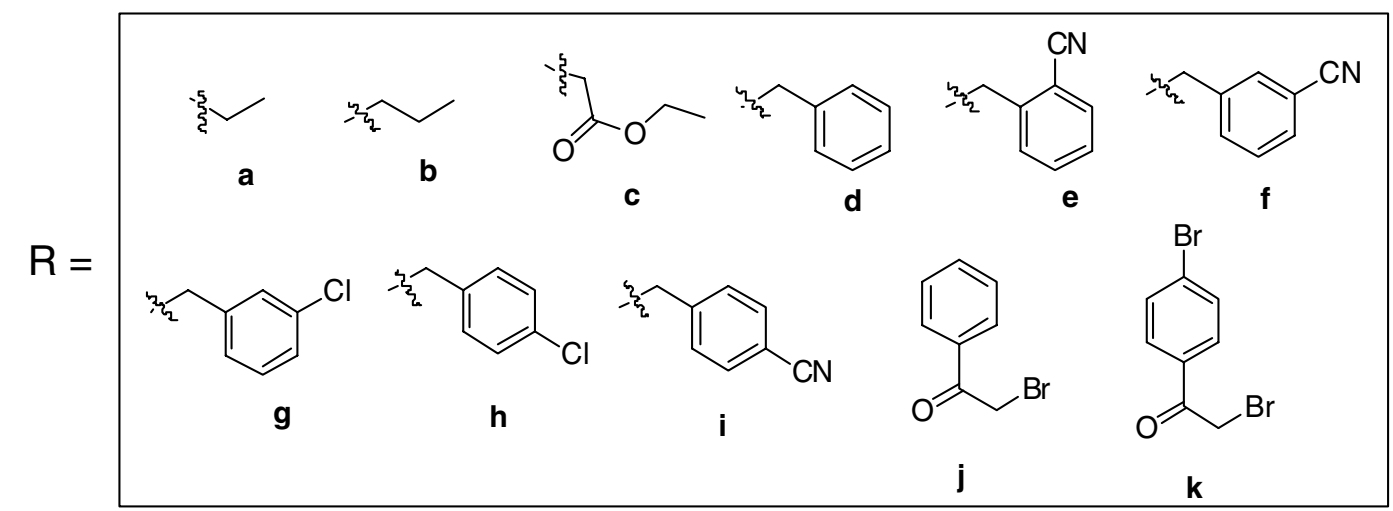

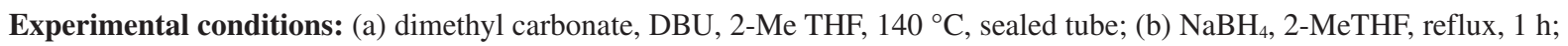

c) $\mathrm{CuBr}, 2$,2'-bipyridine, TEMPO, N-methyl imidazole, acetone, room temperature, $2 \mathrm{~h}$; d) thiazolidinedione, ammonium acetate, acetic acid, toulene, reflux, $3 \mathrm{~h}$; e) $\mathrm{R}-\mathrm{Br} / \mathrm{Ar}-\mathrm{CH}_{2}-\mathrm{Br}, \mathrm{K}_{2} \mathrm{CO}_{3}, \mathrm{DMF}, 80{ }^{\circ} \mathrm{C}, 1-3 \mathrm{~h}$ 
aldehyde $\mathbf{4}$ with thiazolidinedione in presence of ammonium acetate and acetic acid (catalytic quantity) in toluene at reflux for $3 \mathrm{~h}$ gave $(E)$-5-(2-methoxy-6-pentadecylbenzylidene)thiazolidine-2,4-dione (5). Reaction of thiazolidine-2,4-dione (5) with corresponding alkyl bromides, benzyl bromides and phenacyl bromides in presence of potassium carbonate in DMF at $80^{\circ} \mathrm{C}$ for 1-3 h resulted in the formation of corresponding $\mathrm{N}$-alkylated thiazolidine-2,4-dione derivatives 6a-6k. The structures of newly synthesized derivatives 6a-k were determined by ${ }^{1} \mathrm{H}$ NMR, mass and IR spectral data. As a representative example the ${ }^{1} \mathrm{H}$ NMR of ethyl 2-[(E)-5-(2-methoxy-6-pentadecylbenzylidene)2,4-dioxothiazolidin-3-yl]acetate (6c) is discussed here, the proton signals resonating in the aromatic region $7.98 \mathrm{ppm}$ (singlet, $1 \mathrm{H}), 7.22 \mathrm{ppm}$ (double doublet, $1 \mathrm{H}$ ), $6.80 \mathrm{ppm}$ (doublet, $1 \mathrm{H}$ ), $6.70 \mathrm{ppm}$ (doublet, $1 \mathrm{H}$ ) is assigned to the olefin bridge proton (linking AA and thiazolidine ring) and anacardic acid ring protons respectively. The proton signals resonating in the aliphatic region viz., 4.38 ppm (singlet, $2 \mathrm{H}$ ), 3.80 ppm (singlet, $3 \mathrm{H})$ corresponds to the methylene $\left(-\mathrm{CH}_{2}\right)$ and methoxy groups, while the protons resonating at $4.18 \mathrm{ppm}$ (quartet, $2 \mathrm{H}$ ), 2.58 ppm (triplet, $3 \mathrm{H}$ ) and $0.80-1.48 \mathrm{ppm}$ is assigned to ethylester group and anacardic acid side chain protons respectively.

IR interpretation 6c: A strong characteristic band in the region $1732 \mathrm{~cm}^{-1}$ corresponds to $\mathrm{C}=\mathrm{O}$ stretching vibrations of ethyl ester functional group while the peaks in the region 1691 and $1604 \mathrm{~cm}^{-1}$ is assigned to the $\mathrm{C}=\mathrm{O}$ stretching vibrations of thiazolidine ring. The remaining aliphatic and aromatic stretching bands appeared in the expected region.

The ${ }^{13} \mathrm{C}$ NMR of $\mathbf{6 c}$ is in accordance with the desired structure, the signals in the region 168.6, 166.4, 165.6 ppm is assigned to carbonyl signal of ethylester group and 2,4-thiazolidine dione ring, respectively. The remaining carbon signals of the aromatic and aliphatic groups were found to be in the expected region. ESI-MS spectrum carrying a base peak at $m / z 532.5$ $[\mathrm{M}+1]^{+}$is in agreement with the formation of compound $\mathbf{6 c}$. Similarly, the remaining compounds $\mathbf{6 a - k}$ has been fully characterized as per the above description.

Antidiabetic activity: Alloxan administration significantly elevated the fasting blood glucose levels (FBG) levels in all groups. Treatment with thiazolidine-2,4-dione derivatives (6a-k) are continued for 7 days. After the $7^{\text {th }}$ day of treatment, it was observed that some of the significantly reduced the FBG levels when compared to diabetic mice (analyzed by glucometer strips analysis). Out of all synthetic compounds the compounds $\mathbf{6 a}, \mathbf{6 b}, \mathbf{6 c}$ showed significant decrease in FBG levels (when compared to standard drug with insulin) and in terms of hyperglycemic percentage, $60.52,60.06$ and $60.55 \%$, respectively these compounds displayed good antidiabetic activity while the compounds $\mathbf{6} \mathbf{d}, \mathbf{6 j}$ and $\mathbf{6 k}$ displayed moderate antidiabetic activity with $55.15,57.80$ and $57.93 \%$ of hypoglycemic activity. The remaining compounds in the series $\mathbf{6 e - 6 i}$ displayed poor antidiabetic activity.

\section{Conclusion}

We have designed and synthesized some novel 5-(2methoxy-6-pentadecylbenzylidene)-3-alkyl/aryl-substituted thiazolidine-2,4-dione derivatives (6a-6k) utilizing anacardic as starting material. The structural determination of these derivatives have been confirmed by ${ }^{1} \mathrm{H}$ NMR, mass and IR spectro- scopic techniques. Furthermore, these compounds have been evaluated for their antidiabetic property, compounds $\mathbf{6 a}, \mathbf{6 b}$ and $\mathbf{6 c}$ with substitution ethyl, propyl and ethylacetoacetate showed significant decrease in fasting blood glucose levels when compared to standard drug with insulin.

\section{REFERENCES}

1. P.R. Shashikant, K. Prajact, N.S. Dighe, S.B. Bhawar, N. Ana and P. Ashwini, Asian J. Res. Chem., 2, 123 (2009).

2. C.H. Jithendra and P. Muralidharan, Int. J. Green Pharm., 3, 66 (2009); https://doi.org/10.4103/0973-8258.49378.

3. S. Kim, J.J. Lee and D.S. Heo, Biochem. Biophys. Res. Commun., 406, 389 (2011); https://doi.org/10.1016/j.bbrc.2011.02.052

4. N.C. Desai, U.P. Pandit and A. Dodiya, Expert Opin. Ther. Pat., 25, 479 (2015); https://doi.org/10.1517/13543776.2014.1001738

5. O. Bozdag-Dündar, N.D. Evcimen, M. Ceylan-Ünlüsoy, R. Ertan and M. Sarikaya, Med. Chem. Res., 16, 39 (2008);

https://doi.org/10.1007/s00044-007-9008-9.

6. L.R. de Magalhães, I.B.V. Alves, E. dos Santos, V.B.R. da Silva, L.C.A. da Silva, A.L. da Silva, L.C.L. de Oliveira, A.C.N. da Cruz, B.I.M. da Silva, L.F.C. da Costa Leite, M.H. Zaldini, I. da Rocha Pitta, T.G. da Silva and M. do Carmo Alves de Lima, Br. J. Pharm. Res., 4, 1739 (2014); https://doi.org/10.9734/BJPR/2014/11294.

7. D. Patel, P. Kumari and N. Patel, Med. Chem. Res., 21, 2926 (2012); https://doi.org/10.1007/s00044-011-9822-y.

8. M.R. Shiradkar, M. Ghodake, K.G. Bothara, S.B. Bhandari, A. Nikalje and K.C. Akula, ARKIVOC, 58 (2007); https://doi.org/10.3998/ark.5550190.0008.e08.

9. A.M. Ali, G.E. Saber, N.M. Mahfouz, M.A. El-Gendy, A.A. Radwan and M.A. Hamid, Arch. Pharm. Res., 30, 1186 (2007); https://doi.org/10.1007/BF02980259.

10. B.B. Lohray, V. Bhushan, B.P. Rao, G.R. Madhavan, N. Murali, K.N. Rao, A.K. Reddy, B.M. Rajesh, R. Chakrabarti, R.K. Vikramadithyan, P.G. Reddy, R. Rajagopalan, R.N.V.S. Mamidi, H.K. Jajoo and S. Subramaniam, J. Med. Chem., 41, 1619 (1998); https://doi.org/10.1021/jm970444e.

11. C. Prabhakar, G. Madhusudhan, K. Sahadev, C.M. Reddy, M. Sarma, G.O. Reddy, R. Chakrabarti, C.S. Rao, T.D. Kumar and R. Rajagopalan, Bioorg. Med. Chem. Lett., 8, 2725 (1998); https://doi.org/10.1016/S0960-894X(98)00485-5.

12. K.A. Reddy, B. Lohray, V. Bhushan, A.S. Reddy, P.H. Kishore, V.V. Rao, V. Saibaba, A.C. Bajji, B.M. Rajesh, K.V. Reddy, R. Chakrabarti and R. Rajagopalan, Bioorg. Med. Chem. Lett., 8, 999 (1998); https://doi.org/10.1016/S0960-894X(98)00159-0.

13. V. Patil, K. Tilekar, S. Mehendale-Munj, R. Mohan and C. Ramaa, Eur. J. Med. Chem., 45, 4539 (2010); https://doi.org/10.1016/j.ejmech.2010.07.014.

14. C.G. Bonde and N.J. Gaikwad, Bioorg. Med. Chem., 12, 2151 (2004); https://doi.org/10.1016/j.bmc.2004.02.024.

15. A.M. Youssef, M. Sydney White, E.B. Villanueva, I.M. El-Ashmawy and A. Klegeris, Bioorg. Med. Chem., 18, 2019 (2010); https://doi.org/10.1016/j.bmc.2010.01.021.

16. A. Natarajan, Y. Guo, F. Harbinski, Y.-H. Fan, H. Chen, L. Luus, J. Diercks, H. Aktas, M. Chorev and J.A. Halperin, J. Med. Chem., 47, 4979 (2004); https://doi.org/10.1021/jm0496234.

17. H.N. Hafez and A.R. El-Gazzar, Bioorg. Med. Chem. Lett., 19, 4143 (2009); https://doi.org/10.1016/j.bmcl.2009.05.126.

18. T. Tomasic, N. Zidar, M. Mueller-Premru, D. Kikelj and L.P. Masic, Eur. J. Med. Chem., 45, 1667 (2010); https://doi.org/10.1016/j.ejmech.2009.12.030.

19. R.T. Carroll, D.E. Dluzen, H. Stinnett, P.S. Awale, M.O. Funk and W.J. Geldenhuys, Bioorg. Med. Chem. Lett., 21, 4798 (2011); https://doi.org/10.1016/i.bmcl.2011.06.060.

20. N. Sunduru, K. Srivastava, S. Rajakumar, S.K. Puri, J.K. Saxena and P.M.S. Chauhan, Bioorg. Med. Chem. Lett., 19, 2570 (2009); https://doi.org/10.1016/j.bmcl.2009.03.026.

21. Sucheta, S. Tahlan and P.K. Verma, Chem. Cent. J., 11, 130 (2017); https://doi.org/10.1186/s13065-017-0357-2. 
22. M.T. Trevisan, S.B. Pfundstein, R. Haubner, G. Wurtele, B. Spiegelhalder, H. Bartsch and R.W. Owen, Food Chem. Toxicol., 44, 188 (2006); https://doi.org/10.1016/j.fct.2005.06.012.

23. S.S. Mlowe, R.R. Pullabhotla, E.B. Mubofu, F.N. Ngassapa and N.N. Revaprasadu, Int. Nano Lett., 4, 106 (2014); https://doi.org/10.1007/s40089-014-0106-7.

24. S. Mlowe, A.A. Nejo, V.S.R.R. Pullabhotla, E.B. Mubofu, F.N. Ngassapa, P. O'Brien and N. Revaprasadu, Mater. Sci. Semicond. Process., 16, 263 (2013); https://doi.org/10.1016/j.mssp.2012.10.017.

25. M. Misra and U. Aiman, Indian J. Pharmacol., 44, 538 (2012); https://doi.org/10.4103/0253-7613.99348.
26. D. Kumar, S. Kumar, S. Kohli, R. Arya and J. Gupta, Asian Pac. J. Trop. Med., 4, 900 (2011); https://doi.org/10.1016/S1995-7645(11)60215-0.

27. P.V. Swamy, K.B. Chandrasekhar and K.C. Pullaiah, China J. Appl. Chem., 32, 492 (2015).

28. W.-C. Shieh, S. Dell and O. Repic, J. Org. Chem., 67, 2188 (2002); https://doi.org/10.1021/jo011036s.

29. N.J. Hill, J.M. Hoover and S.S. Stahl, J. Chem. Educ., 90, 102 (2013); https://doi.org/10.1021/ed300368q. 